\title{
2015 London Declaration on Person- and People-centered Primary Care and Public Health
}

\author{
Emerging from the Third International Congress of Person Centered Medicine, London, October 31, 2015 and \\ released by the International College of Person Centered Medicine on December 1, 2015.
}

Sustainable improvement in the health and well being of the people of all nations will be achieved with a shared vision, understanding and action through the integration of primary care and public health. This should contribute to access to and coverage for universal health care services.

Public health is the science and art of promoting health, preventing disease and prolonging life (adding life to years and years to life) through the organized efforts of society. Public health needs to be person- and people- centered taking into account the biomedical, social, cultural, psychological, and spiritual elements that are crucial to understanding the whole person and the community at large.

Primary care by its very nature is person-centered (rather than disease-centered) along the person's life, team-based (with partnerships at multiple levels), and integrative of all relevant individuals, groups and sectors. Primary care through trusted and healing relationships with people over time can start to achieve the required integration and coordination of care that leads to a better health care experience, improved health outcomes and lower cost. It is essential to empower and engage people in their care through shared decision-making.

Primary care and public health each play critical roles in tackling the complex health problems that exist internationally, nationally and locally. Their shared goal of health improvement will encourage and maintain partnerships between the different health and social sectors designed to bring about sustained improvements in population health.

Person centered health care is based on the underlying value system of medical ethics. Trust between a person and a physician/health care professional is fundamental. Likewise, public health physicians and practitioners should have a commitment to equity, social justice and sustainable development, and recognize the importance of the health of the community as well as of the individual. They should respect diversity, self-determination, empowerment and community participation. A person-centered approach supports the freedom and the responsibility to develop a person's life in ways that are personally meaningful and that are respectful of others and the environment where all live together.

For progress towards the integration of health services there needs to be a shared goal of population health improvement and community engagement. Defining and addressing population health needs are shared responsibilities of all stakeholders. Leadership is required at multiple levels that bridges disciplines, programs, and agencies. This may help reduce fragmentation of care, manage change, ensure accountability, clarify roles and foster continuity and interdisciplinary communication and collaboration. The establishment of a shared infrastructure and settings is key to sustainability, for enduring value and impact; and for the sharing and collaborative use of data and analysis. The accurate and secure recording of health information within primary care and public health is essential.

\section{Call to Action}

The International College of Person Centered Medicine, in response to the above considerations and following discussions at its Third International Congress in London, issues the following call to action.

1. Establish a shared goal of improvement in the health and well being of the population through person- and peoplecentered primary care and public health.

2. All public health and primary care professionals should share a core set of attitudes and values integral to their ethical responsibilities to individuals and communities.

3. Both primary care and public health professionals should be educated in part through shared curricular programs and educational experiences aimed at enhancing communication and collaboration.

4. The whole person of primary care and public health professionals should be recognized, as they are asked to enhance and create links and relationships with each other and all stakeholders. 
5. Every health system should be based on universal access to person- and community-centered primary care. Thus, primary care should be delivered within the social and occupational networks of each person.

6. Prevention and health promotion public health programs, with due attention to environmental concerns, should be integrated with primary care.

7. Identify available data bases and create new ones that ensure personal confidentiality and are interactive to facilitate the planning of both primary care and public health.

8. Establish common research networks to foster and support the integration of primary care and public health to improve population health and the use of healthcare resources in a person-centered manner, as illustrated by patient outcome and experience reports.

9. Develop pilot programs that better integrate primary care and public health and other health and social services to address effectively broader social determinants of health.

10. The integration of health and social care should recognize that both are part of complex adaptive systems with many components that reciprocally influence one another in dynamic ways. 